\title{
Observatorio
}

\section{Arquitectura de la información en entornos web}

\author{
Por Mario Pérez-Montoro
}

Resumen: La arquitectura de la información, partiendo de los sólidos principios clásicos de la ciencia de la información tradicional, nace hacia finales de los años 90. Es una disciplina encargada de estructurar, organizar y etiquetar los elementos que conforman los entornos informacionales para facilitar la búsqueda y recuperación de la información que contienen y mejorar, así, la utilidad y el aprovechamiento de la misma por parte de sus usuarios. Entre los principales sistemas o estructuras que conforman la anatomía arquitectónica de un sitio web destacan los sistemas de organización, de etiquetado, de navegación, de bús-

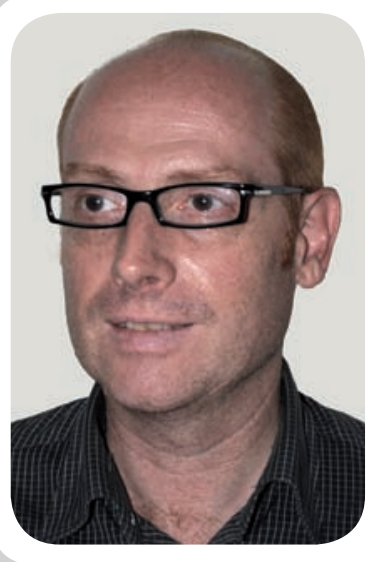

Mario Pérez-Montoro es doctor en filosofía y ciencias de la educación por la Universitat de Barcelona (UB) y máster en organización de sistemas de documentación en la empresa por la Universitat Politècnica de Catalunya. Ha realizado estudios de postgrado en el Istituto di Discipline della Comunicazione de la Università di Bologna (Italia) y ha sido profesor visitante del CSLI (Center for the Study of Language and Information) de la Stanford University (California, EUA). Es profesor de la Facultat de Biblioteconomia i Documentació de la UB. Su investigación se centra sobre algunos aspectos (conceptuales, semánticos, epistemológicos y pragmáticos) relacionados con la gestión de la información y del conocimiento. Es autor del libro Arquitectura de la información en entornos web (Trea, 2010).

queda y los vocabularios controlados. Respecto a su praxis, la elaboración de la anatomía arquitectónica de un sitio web se centra en los aspectos relacionados con las necesidades de sus usuarios tipo.

Palabras clave: Arquitectura de la información, Sistemas de organización, Sistemas de etiquetado, Sistemas de navegación, Sistemas de búsqueda, Vocabularios controlados, Metodología.

\section{Title: Information architecture in the web environment}

Abstract: Information architecture (IA), which is based on the classical principles of solid traditional Information Science, was born in the late '90. This discipline deals with structuring, organizing and tagging elements of informational environments to facilitate searching and retrieval of information they contain, thereby improving the usefulness and applications of IA. Main systems or structures that build a website's architectonic anatomy are the organization systems, labeling systems, navigation systems, search systems and controlled vocabularies. Nowadays, the information architecture praxis and design is centered on user needs.

Keywords: Information architecture, Organization systems, Labeling systems, Navigation systems, Search systems, Controlled vocabularies, Methodology.

Pérez-Montoro, Mario. “Arquitectura de la información en entornos web”. El profesional de la información, 2010, julioagosto, v. 19, n. 4, pp. 333-337.

DOI: $10.3145 /$ epi.2010.jul.01

\section{Introducción}

A ESTAS ALTURAS NADIE pone en duda que internet es uno de los fenómenos más importantes que se han producido en toda la historia de la humanidad y que ha transformado, en el sentido más amplio y profundo, la comunicación humana.

Cada día que pasa millones de nuevas páginas web se añaden a las ya existentes y se muestran en internet para ser visitadas. Su éxito es sin embargo muy dispar. Algunas cubren sus objetivos comunicativos recibiendo millones de visitas y otras pasan desapercibidas sin apenas registrar usuarios que se interesen por ellas.
Las causas que pueden explicar esa variación en el éxito comunicativo de las webs pueden ser múltiples: el grado de interés de sus contenidos, el posicionamiento al que las sitúan los buscadores en los resultados de las búsquedas, o su nivel de usabilidad y accesibilidad.

Todas estas causas, por sí mismas o de forma combinada, pueden explicar el éxito comunicativo de un sitio web. Sin embargo existe otra causa diferente que destaca por su poder explicativo y porque en muchas ocasiones puede justificar ese éxito o fracaso: la utilidad de la propia web. En este contexto debemos entender esa utilidad como la capacidad que tiene una web de permitir que el visitante encuentre aquella parte de 
la información que está buscando y que ella aloja. Si una persona, debido a la estructura del sitio web, no es capaz de encontrar lo que busca aunque el sitio lo albergue, muy probablemente abandonará esa web y no la utilizará más. Si una web no es útil seguramente estará condenada al fracaso comunicativo.

La disciplina que se encarga de diseñar los sitios web para que sean útiles - para que el usuario pueda encontrar lo que busca, si se halla en el sitio- se denomina arquitectura de la información (IA).

Partiendo de los sólidos principios clásicos de la ciencia de la información tradicional (principalmente de la disciplina de la organización y la representación del conocimiento), la IA nació hacia finales de los años 90 (Morrogh, 2002; Wurman, 1997). En términos generales podemos convenir que se trata de una disciplina encargada de estructurar, organizar y etiquetar los elementos que conforman los entornos informacionales para facilitar de esta manera la localización (y el acceso) a la información contenida en los mismos y mejorar así su utilidad y aprovechamiento por parte de sus usuarios (Pérez-Montoro, 2010).

"La IA se encarga de estructurar, organizar y etiquetar los elementos de información para facilitar su localización"

\section{Anatomía arquitectónica}

La arquitectura de la información de un sitio web se caracteriza en muchos de sus elementos por no ser directamente observable por parte del usuario. En cierta manera es una estructura invisible que se encuentra por debajo o por detrás de la fina superficie que puede apreciar el visitante cuando interacciona con las páginas web.

Existe una serie de sistemas o estructuras no observables que definen la arquitectura de la información a los que se denomina "componentes de la IA" o "estructuras arquitectónicas" de una web, o también "anatomía de la IA" (Morville; Rosenfeld, 2006).

Los principales componentes de la IA que suelen identificarse son los siguientes: sistemas de organización, de etiquetado, de navegación, de búsqueda, y vocabularios o lenguajes documentales.

Los componentes arquitectónicos que conforman normalmente un sitio web presentan algunas características comunes. Por un lado están diseñados para permitir descubrir y recuperar la información relevante que contiene el sitio.
Por otro lado no actúan de forma autónoma sino que lo hacen de forma articulada (Wodtke, 2002). Y esa articulación es tan estrecha que a veces es difícil decidir dónde acaba uno de los sistemas y dónde empieza otro. Así por ejemplo, el sistema de búsqueda se diseñará teniendo en cuenta alguna de las decisiones básicas tomadas a la hora de implementar el sistema de navegación, el de etiquetado o los lenguajes controlados utilizados para representar los contenidos de la web.

Por último, los componentes no pueden ser fijos e inmutables, como lo podrían ser las vigas de un edificio en el caso de la arquitectura tradicional. Deben interaccionar entre ellos, expresando las relaciones conceptuales que mantienen los contenidos que alberga la web, y adaptándose continuamente a los cambios que éstos vayan presentando en el tiempo.

\section{"El sistema de etiquetado determina los términos utilizados para nombrar las categorías, opciones y links utilizados"}

\section{Sistemas de organización}

Se encargan de estructurar y organizar los contenidos de un sitio web. Acostumbran a construirse mediante la clasificación, a partir de uno o varios criterios concretos (por ejemplo el tema sobre el que versan, la fecha de creación o la audiencia a la cual van dirigidos), de los contenidos que alberga esa página.

Suelen encontrarse formados por dos componentes: los esquemas de organización y las estructuras de organización.

Los esquemas de organización dividen y clasifican los ítems de información o contenidos que alberga un sitio web en grupos a partir de un criterio. Los ítems de cada grupo comparten unas características definitorias que pueden ser consideradas como el aspecto crítico del esquema. Los criterios más utilizados son los alfabéticos, los cronológicos y los temáticos.

Por otro lado, las estructuras de organización sistematizan los grupos de ítems de información o contenidos resultantes de los esquemas, mostrando las dependencias lógicas que existen entre estos grupos. Dependencias que deben ser consideradas como el aspecto crítico de la estructura.

\section{Sistemas de etiquetado}

Definen y determinan de una forma consistente y eficiente los términos utilizados para nombrar las ca- 


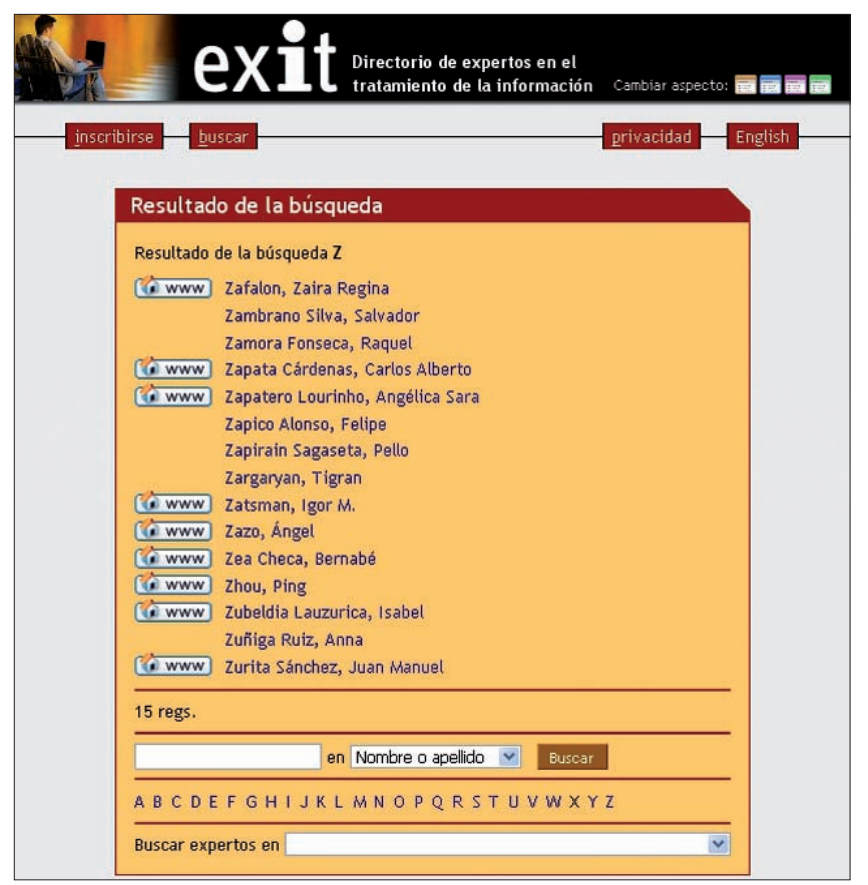

Figura 1. Esquema de organización alfabético en el directorio Exit http://www.directorioexit.info

\section{"Los componentes de la IA de un sitio web son: sistemas de organización, etiquetado, navegación, búsqueda, y vocabularios o lenguajes documentales"}

tegorías, opciones y links utilizados en la web con un lenguaje lo más útil posible para los usuarios.

El etiquetado permite tener presentes los ítems sin que físicamente se encuentren visibles, sólo se ven sus nombres. Al conocer cuántos ítems hay se tiene una idea del consumo de espacio físico en la web, así como del espacio cognitivo (por así decirlo) en el cerebro del usuario. O sea, es lo mismo que nos da un índice, que nos ofrece la extensión física y la temática.

El uso de etiquetas permite no tener que ofrecer simultánea y literalmente todos los contenidos en el mismo nivel o espacio, consiguiéndose aligerar visualmente la página web. El usuario decide si obtiene o no la información representada clicando o no en la etiqueta.

Las etiquetas se presentan en dos formatos: el icónico y el textual, según usen imágenes o iconos, o términos o palabras como etiquetas para representar contenidos.

\section{"Los sitios web se vertebran mediante esquemas y estructuras de organización"}

\section{Sistemas de navegación}

Nos permiten como visitantes movernos de una forma cómoda por las diferentes secciones y páginas que componen un sitio web. Mediante la utilización de ciertos recursos de visualización, ofrecen un método de orientación para que los usuarios puedan acceder a los contenidos del sito web sabiendo en cada momento dónde se encuentran, de dónde vienen y hacia dónde pueden ir dentro de la estructura de esa web.

Los principales tipos de sistemas de navegación que podemos encontrar en una página web son: sistemas constantes, locales y contextuales.

Los sistemas constantes (o globales) de navegación suelen encontrarse incorporados en la mayoría de páginas, en forma de una barra de navegación horizontal en la parte superior. Permiten que el usuario se oriente y sepa en todo momento dónde se encuentra y dónde puede ir. Acostumbran a permitir el acceso a las páginas o contenidos clave o más importantes del sitio web haciendo clic en partes de la barra. No suelen reflejar la jerarquía estructural entre los contenidos concretos de una página, aunque sí ofrecen una idea de cómo se encuentra estructurado el sitio web completo. Como este tipo de sistemas tienen consecuencias directas sobre la usabilidad del sitio en el que se implantan, acostumbran a ser sometidos a numerosos tests y puestos a prueba antes de incorporarlos definitivamente.

Figura 2. Combinación de etiquetado icónico y textual en la web de Aspirina http://www.aspirina.com 


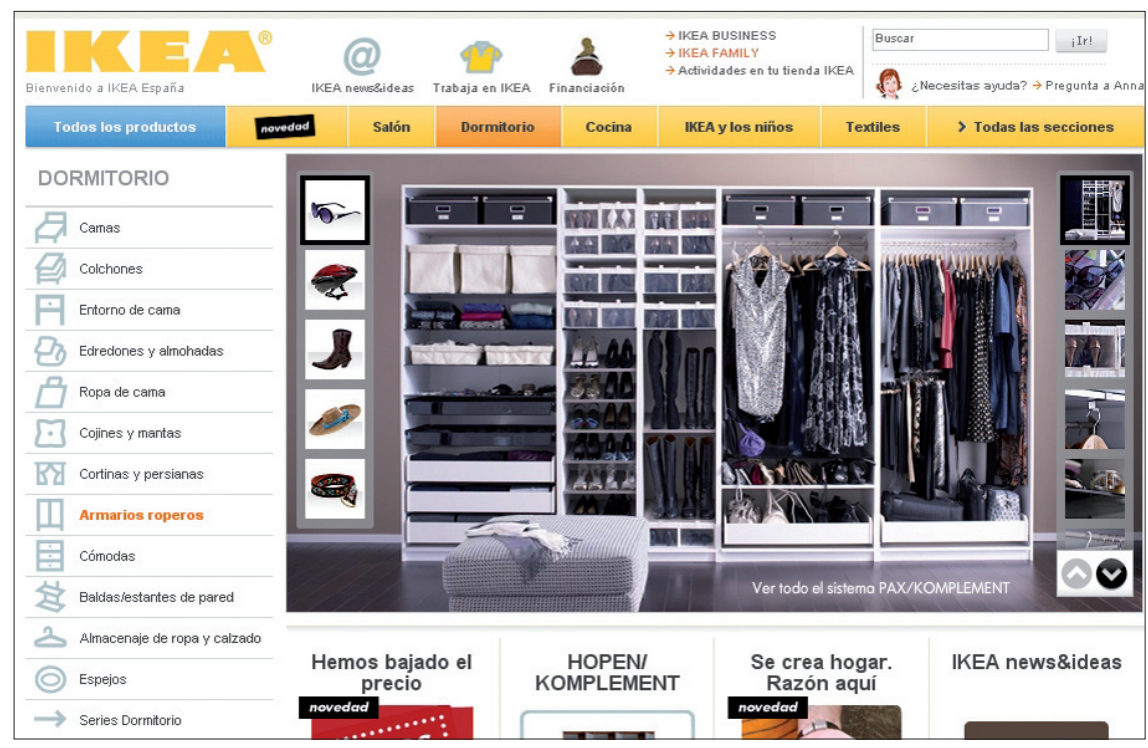

Figura 3. Sistema constante de navegación (barra horizontal superior) y sistema local de navegación (menú en columna izquierda) de la página de lkea http://www.ikea.com

Los sistemas locales de navegación son recursos compuestos normalmente por una barra vertical (aunque en algunas ocasiones se diseña con forma horizontal) de navegación que complementan los anteriores sistemas constantes y sirven para explorar una página concreta. También orientan al usuario para que sepa qué hay cerca de la página visitada y dónde puede ir.

Existen ciertas relaciones entre contenidos que se escapan o que no pueden ser recogidas por los sistemas constantes y locales descritos. Por ello, los sistemas contextuales de navegación permiten relacionar un contenido que se está visitando con otro contenido en otra página mediante un enlace o hipervínculo ligado a un literal o icono.

\section{"Con el sistema de navegación podemos movernos por las diferentes secciones y páginas de un sitio web"}

\section{Sistemas de búsqueda}

Previa una indización de páginas y objetos del sitio web, tales sistemas posibilitan la búsqueda y recuperación de la información dentro del mismo. La interfaz es una de las piezas clave que conforman un sistema de búsqueda en el contexto de una web pues es el punto donde se produce la interacción entre el usuario y el propio sistema de búsqueda.

Por otro lado, su importancia queda más reforzada si somos conscientes de que, de acuerdo con el grado de alfabetización informacional que presenten, la mitad de los usuarios que visitan una página web acostumbran a comenzar la interacción con la misma desde una sesión de búsqueda.

Cuando un usuario propone una ecuación de búsqueda a un sistema, éste le ofrece un conjunto de resultados que son una representación de los contenidos que se adecuan más a la demanda de información del usuario.

En esta misma línea, siempre que se intenta configurar la presentación y visualización de resultados provenientes de un proceso de búsqueda se suelen tener en cuenta dos aspectos críticos para su correcta implementación: el equilibrio entre la información mostrada por resultado y la cantidad total de resultados recuperados.

\section{Vocabularios controlados}

El último de los elementos que conforman la anatomía arquitectónica de una página web son los vocabularios controlados o lenguajes documentales. Son recursos documentales (tesauros, taxonomías, anillos de sinónimos, etc.), invisibles en su totalidad para los usuarios y visitantes de la página en la mayoría de los casos, que facilitan la búsqueda y recuperación de información.

La planificación y construcción de los lenguajes documentales se sustenta sobre dos operaciones básicas: la indización y la clasificación. En un sentido lato la indización es una operación en la que se asigna a cada documento (a cada contenido, en el contexto web) una serie de términos que representan el tema o temas de ese documento. Por otro lado la clasificación es una operación en la que se asigna a cada documento o contenido un único término que representa el tema principal de ese documento.

La información resultante de la indización y la clasificación suele incorporarse operativamente al contenido o página web mediante los metadatos asociados a esa página para que funcione articulando los sistemas de organización, etiquetado, navegación y búsqueda.

En un sentido técnico, los metadatos son un conjunto de etiquetas (de metaetiquetas, estrictamente hablando) que describen una página web y que se encuentran situados en su cabecera (generalmente invisible de forma directa para el usuario).

Los metadatos describen la página web con sus contenidos, y los correspondientes formatos. Normalmente se utiliza algún tipo de estándar para realizar esa 


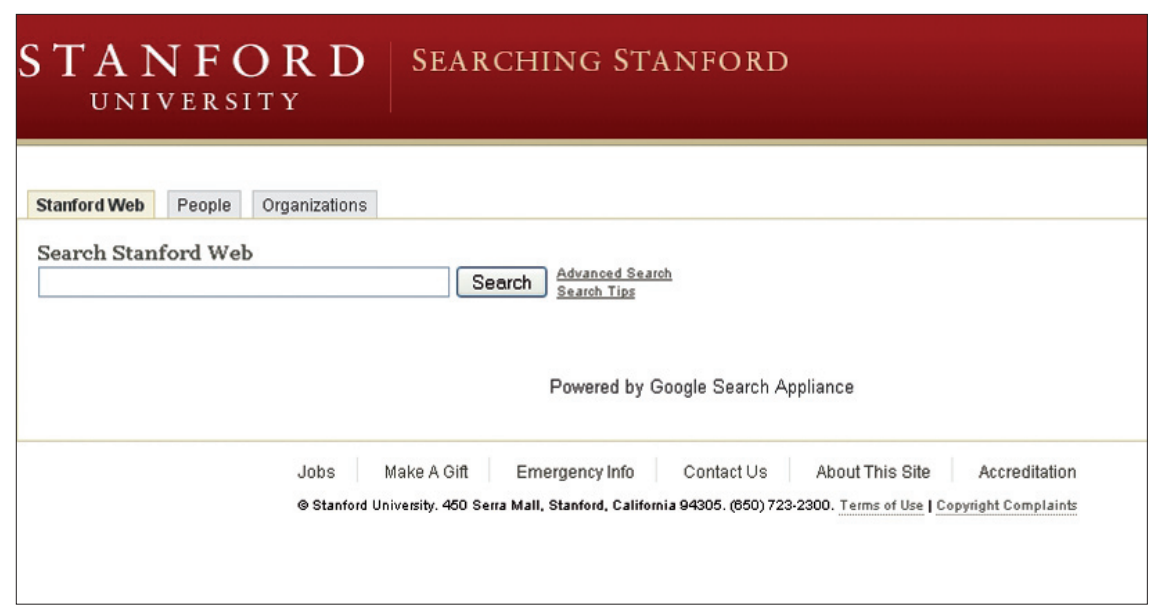

Figura 4. Sistema de búsqueda de la web de la Stanford University http://www.stanford.edu

descripción. En el caso de los recursos de información general, el estándar internacional Dublin Core es uno de los más habituales según el cual la descripción de una página web debe incluir: información sobre su contenido (título, materia, descripción, lengua, etc.), sobre la propiedad intelectual de la misma (autor, editor, derechos, etc.), sobre su temporalidad (fecha de creación, de modificación, etc.) y sobre el contexto de la página (dónde está ubicada dentro del sitio web o con qué otras páginas o contenidos está asociada, por ejemplo).

\section{"Los vocabularios controlados, invisibles para los usuarios, facilitan la búsqueda y recuperación de información"}

\section{Metodología para la IA}

Desde el inicio de internet y hasta hace no muchos años, en el diseño de los sitios web sólo había un proceso importante y sobre el que se centraban todos los esfuerzos y atenciones: la elaboración del código html de la página. Sin casi ningún tipo de análisis, investigación o planificación previa, se acostumbraba a implementar directamente la página web.

Esa primera actitud o estrategia está siendo desterrada. En su lugar se va pasando, de atender de forma exclusiva los aspectos técnicos de la elaboración de la página web, a diseñar ese sitio web y por tanto la anatomía de su IA, pensando en las necesidades de sus usuarios tipo (Garrett, 2002). El resultado de ese cambio es obvio: se consiguen diseñar páginas web que son mucho más útiles para esos usuarios, que terminarán interaccionando con las mismas y satisfaciendo sus necesidades de información. quitectónicas. Gijón: Trea, 2010.
No existe un estándar metodológico ampliamente consolidado y compartido por el grueso de los profesionales. Sin embargo, para llevar a cabo el diseño e implementación centrada en el usuario de la arquitectura de la información de un sitio web, se suelen cubrir las fases de análisis, diseño e implantación.

En la primera, análisis (o investigación), se determina el escenario general. Los estudios que suelen incluirse en esta fase son un análisis del contexto, un análisis del contenido y un análisis de los usuarios.

A partir de la información recabada en el análisis, en el diseño se decide y representa de forma exhaustiva y pormenorizada la IA del sitio web. En este estadio se suele llevar a cabo el inventario y los modelos de los contenidos, la representación del vocabulario, el prototipado y la evaluación de la propuesta arquitectónica.

En la implantación, la propuesta de IA se plasma con el prototipado resultante de la fase de diseño. Es el momento en el que, a partir de los resultados de las dos fases anteriores, se realiza la comunicación de la propuesta arquitectónica, se contribuye en la puesta en marcha del sitio web y se crean sus guías de estilo ar-

\section{"Se observa cómo aumenta el interés por el diseño de los sitios web pensando en las necesidades de sus usuarios"}

\section{Bibliografía citada}

Garrett, Jesse-James. The elements of user experience: user-centered design for the web. Indianapolis: New Riders Publishing, 2002.

Morrogh, Earl. Information architecture: an emerging $21^{\text {st }}$ century profession. Upper Saddle River, NJ: Prentice Hall, 2002.

Morville, Peter; Rosenfeld, Louis. Information architecture for the World Wide Web. $3^{\text {rd }}$ edition. Sebastopol (CA): O’Reilly Media Inc., 2006.

Pérez-Montoro, Mario. Arquitectura de la información en entornos web.

Wodtke, Cristina. Information architecture: blueprints for the Web. Boston: New Riders Publishing, 2002.

Wurman, Richard. Information architects. New York: Graphis Inc., 1997.

Mario Pérez-Montoro. Departament de Biblioteconomia i Documentació, Universitat de Barcelona.

perez-montoro@ub.edu 


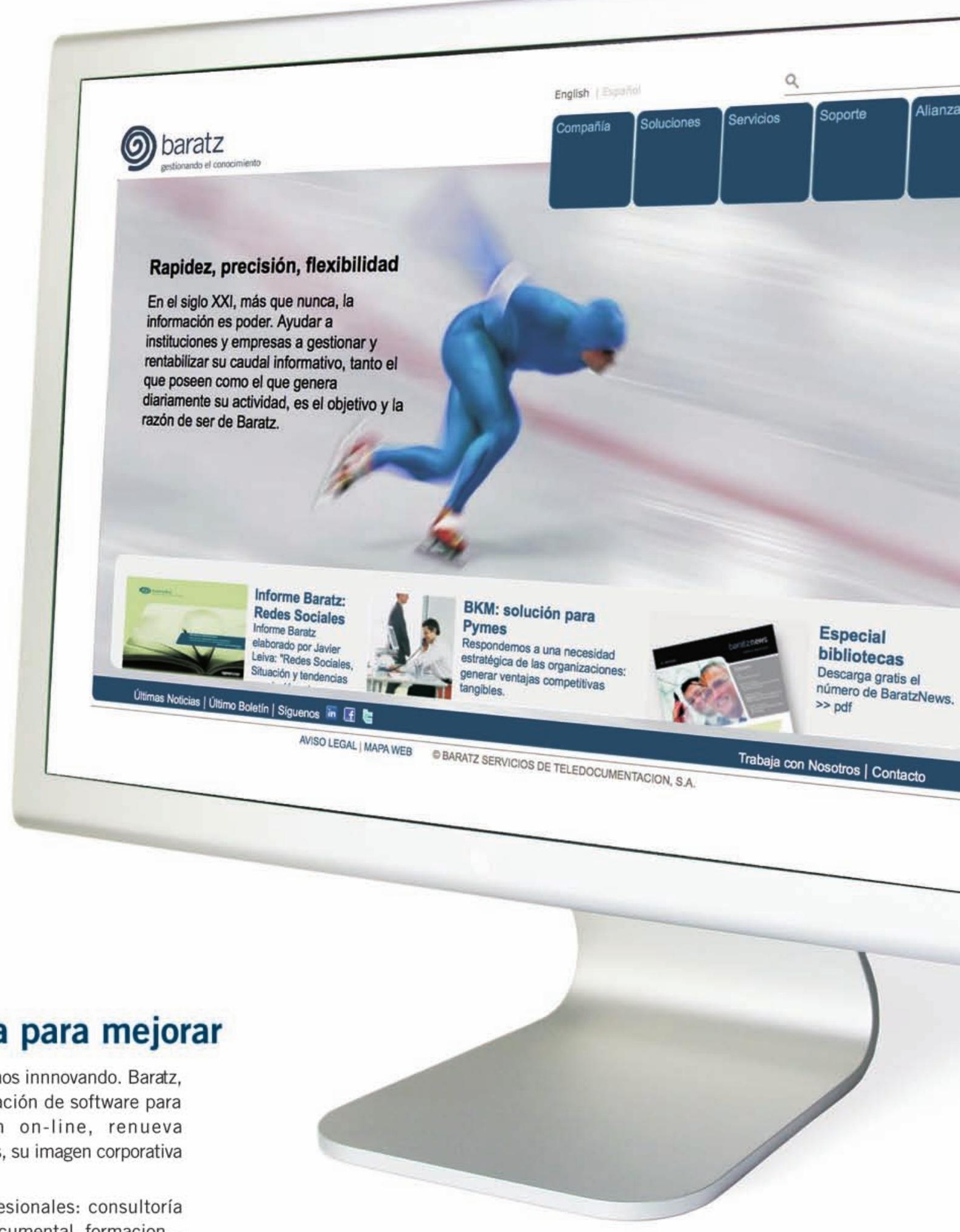

\section{Baratz cambia para mejorar}

Después de 25 años seguimos innnovando. Baratz, empresa pionera en la creación de software para acceder a información on-line, renueva completamente sus servicios, su imagen corporativa y también su web.

- Nuevos servicios profesionales: consultoría documental, gestión documental, formacion...

- Nuevos productos: Absys.edu, AbsysNOVA, DigiNet y AlbaláNET.

Todo Baratz está a una tecla: www.baratz.es

\section{0 informa@baratz.es}

